\title{
UNE MÉTHODE RAPIDE DE LA PHOSPHOMONOESTÉRASE POUR LE CONTROLE DE LA PASTEURISATION DU LAIT (1)
}

\author{
par HARRY SCHARER \\ Chemical Laboratory, Department of Health, New York City.
}

Malgré que les autorités sanitaires ont défini dans leurs ordonnances quelles sont les conditions requises pour la pasteurisation, elles se trouvent fréquemment devant des cas d'infraction à leurs règlements à ce sujet. Il est impossible d'exercer un contrôle satisfaisant, par l'inspection des usines, sur l'élément humain et les nombreux facteurs mécaniques qui peuvent intervenir pour rendre la pasteurisation défectueuse. Entre les visites des inspecteurs des usines, des appareils enregistreurs de la température peuvent se dérégler, ne plus fonctionner, e certains défauts de fonctionnement des appareils ne sont perceptibles que pour les inspeateurs, qui ont une connaissance approfondie des nombreux systèmes de pasteurisation, et des outillages parfois compliqués, qui sont actuellement utilisés; d'autres irrégularités ne peuvent être décelées uniquement par la vue.

Le Code sanitaire de la ville de New-York exige que dans la pasteurisation lente (holding system), la plus répandue dans ce pays, le lait soit chauffé à une température d'au moins $143^{\circ} \mathrm{F}$. (6107 C.), qu'il soit maintenu à cette température pendant 30 minutes et ensuite rapidement refroidi. Fréquemment, on n'a aucune garantie concernant la température et la durée du chauffage. De la mousse insuffisamment chauffée peut avoir été mélangée à du lait réglementairement pasteurisé [1]. Lorsqu'il n'y a pas eu d'agitation du lait dans le réservoir, au cours du chauffage, il y a toujours des risques qu'il y ait eu une répartition inégale de la température. A ce sujet, nous mentionnons la définition de l'Etat de New-York (Code sanitaire, art. 22) :

"Ni lait, ni crème ne seront désignés, ni par éorit, ni verbalement, comıme pasteurisés, si toute particule de ce lait ou de cette crème n'a pas été soumise, sans discontinuation, pendant 30 minutes, à une température de $143^{\circ} \mathrm{F}$. ( $61^{\circ} 7 \mathrm{C}$.) ou supérieure. »

La définition fédérale insiste également sur " toute particule ".

Les épreuves chimiques proposées jusqu'ici pour déceler la pasteurisation défectueuse ou inefficace du lait peuvent être groupées

(1) Journal of Dairy Science, vol. XXI, janvier 1938, p. 21 (Traduction R. N. Göransson). 
de la façon suivante, selon le principe sur lequel elles sont basées.

1. L'examen microscopique : L'épreuve cellulaire de Frost [2].

2. Les propriétés physiques : L'épreuve de la montée de la crème [3], la turbidité [4], la coagulation [5], la lactalbumine [6], les suspensions [7].

3. L'activité des enzymes : La peroxydase [8], la catalase [9], l'aldéhyderéductase [11], l'amylase $[8,12,13]$ et la phosphatase [14].

Lors de l'adoption de la pasteurisation à basse température, plusieurs de ces épreuves sont devenues inapplicables ou inefficaces $[6,8,12,15,16,17,18,19,21,22,23]$. Dans presque toutes les méthodes, jusqu'ici connues, paraît intervenir un facteur personnel ou un autre facteur, qui les rend susceptibles de la critique d'inexactitude, lors de leur application par d'autres que ceux qui les ont préconisés. Au début de 1935, l'Auteur de ces lignes élabora une épreuve modifiée de l'amylase, qui, dans quelques milliers d'applications, permit de déceler du lait oru, indiqua l'addition de lait oru, dans la proportion de $5 \%$, à du lait chauffé, ou une période de chanffage de 15 minutes ou moins, ou une température de chauffage du lait de $140^{\circ} \mathrm{F}$. $\left(60^{\circ} \mathrm{C}\right.$.) ou inférieure. Les résultats obtenus avec cette méthode modifiée étaient encourageants et indiquèrent qu'une méthode plus sensible était nécessaire.

L'épreuve de la phosphatase récemment préconisée présente une plus grande sensibilité, mais elle exige trop de temps et est trop compliquée pour l'application dans la pratique courante. KAY et Graham déclarèrent que pour les températures de pasteurisation américaines $\left(142^{\circ} \pm 2^{\circ} 5 \mathrm{~F} .=61^{\circ} 1 \pm 1^{\circ} 1 \mathrm{C}\right.$.) une durée d'incubation de 2 heures et demie était suffisante, mais GILcreas et Davis [20] affirmèrent que dans l'épreuve quantitative il fallait 24 heures pour des délimitations nettes. Les données obtenues dans ce laboratoire confirment ces affirmations. En dehors de cela, la préparation du réactif de FoLIN et Ciocalteu demande des soins minutieux et exige assez de temps ; en plus, il présente un défaut de spécificité : il réagit avec nombre de phénols, dans lesquels il y a substitution du groupe hydroxyle et même avec des composés stanneux et ferreux [24, 25].

$\mathrm{Au}$ mois de janvier 1937, furent faites des recherches sur l'action des réactifs précipitant les protéines en conjonction avec d'autres réactifs phénolés et il fut constaté que l'acétate basique de plomb utilisé avec 2,6 de dibromoquinonechloroimide donnait entière satisfaction. La sensibilité de ce réactif vis-à-vis du phénol est telle, qu'il décèle sa présence dans la proportion d'une partie pour vingt millions, et il ne réagit qu'avec des phénols, dans lesquels il n'y a 
pas eu substitution du groupe hydroxyle, en donnant lieu à une coloration bleue, sur laquelle on peut se baser pour une estimation quantitative des phénols [26]. Dans ce mémoire, est décrite une technique qui permet l'utilisation de ce réactif, qui ne demande pas beaucoup de temps, est peu onéreuse, n'exige d'autre outillage que celui qu'on peut trouver dans n'importe quel laboratoire officiel ou industriel et dans laquelle le facteur personnel n'a que peu d'importance. Malgré le nombre limité de déterminations (environ 10.000), la concordance des données est telle que la méthode paraît digne d'études ultérieures et détaillées.

Cette méthode est basée sur la destruction par le chauffage de la phosphomonoestérase (1) (constituant, toujours présent dans le lait oru, dont $96 \%$ sont détruits par le chauffage à la température de $143^{\circ} \mathrm{F}$. ( $61^{\circ} 7 \mathrm{C}$.) pendant 30 minutes [14]), et la faculté de cette enzyme d'hydrolyser le phénylphosphate disodique [27] avec mise en liberté de phénol, qui est estimé colorimétriquement avec le 2,6 dibromoquinonechloroimide. L'épreuve est faite en milieu alcalin à un $p \mathrm{H}$. d'environ 9,6 , qui est optimum pour le développement de la coloration. A un $p \mathrm{H}$ moins élevé, le développement de la coloration est indûment prolongé, tandis qu'à un $p H$ plus élevé il peut faire défaut [26]. La rapidité du développement de la coloration est en raison directe de la quantité de phénol présente ; un excès de l'imide active également la formation d'indophénol, mais un trop grand excès avec de petites quantités de phénol ajoute du jaune au bleu.

\section{MÉTHODE MODIFIÉE DE LA PHOSPHATASE}

\section{Réactifs}

2,6 Dibromoquinonechloroimide (désigné ei-dessous par $\mathrm{BQC}$ ) (Eastman Kodak Chemical, No 2304).

Dissoudre 0 gr. 04 dans $10 \mathrm{~cm}^{3}$ d'aleool éthylique à $95 \%$. Dans des flacons bien bouchés, cette solution peut être conservée plusieurs jours. Il fut jugé opportun de conserver cette solution dans un flacon compte-goutte d'Owen, en verre d'une couleur verte de $1 / 4$ once ( 7 gr. 09) (modèle $\mathrm{n}^{0}$ 90943). Ce flacon débite un centimètre cube de la solution alcoolique d'imide en 50 gouttes. Deux gouttes ou $1 / 25$ de centimètre cube de la solution contiennent 0 mgr. 16 d'imide. Cette quantité doit être considérée comme un excès adéquat.

(1) Follex et KAy $[28,29,32]$ classent les phosphatases, désignant comme phosphomonoestérase alcaline la phosphatase du lait, qui est active à un $p$ H alcalin. A ce point de vue, il a paru opportun d'insister sur cette dénomination en vue de diffé. rencier les caractéristiques de cette enzyme de celles des autres phosphatases. 
Acétate basique de plomb (préparé par la méthode de Horne).

Indications A.C.S. (American Chemical Society).

Faire bouillir 280 grammes d'acétate basique de plomb sec avec $500 \mathrm{~cm}^{3}$ d'eau pendant 5-10 minutes. Refroidir, laisser déposer, filtrer et diluer jusqu'à $500 \mathrm{~cm}^{3}$.

\section{Solution Tampon boratée.}

Dissolvez 28 gr. 427 de $\mathrm{Na}^{2} \mathrm{~B}^{4} \mathrm{O}^{7} \cdot \mathrm{IOH}^{2} \mathrm{O}$ (qualité pharmaceutique) dans $900 \mathrm{~cm}^{3}$ d'eau distillée, en agitant vigoureusement pour prévenir la formation de grumeaux. Ajoutez $3 \mathrm{gr}$. $27 \mathrm{de} \mathrm{NaOH}$ sous forme d'une solution concentrée ( 2 à $5 \mathrm{~N}$.), refroidir et diluer jusqu'à un litre.

\section{Substrat-Tampon.}

Ii fut constaté que le phénylphosphate disodique fourni par les "British Drug Houses Ltd." contenait du phénol libre dans des proportions notables (1); de ce fait, le sel fut lavé au moyen d'éther éthylique, jusqu'à l'élimination complète du phénol, démontrée par une réaction négative vis-à-vis du phénol. Dans ce but, on additionne $10 \mathrm{~cm}^{3}$ d'eau distillée à $100 \mathrm{~cm}^{3}$ de l'éther de lavage, on évapore l'éther, puis on ajoute $1 / 2$ centimètre cube de la solution tampon boratée, ensuite 4 gouttes de BQC. Le développement d'une coloration bleue indique la présence de phénol.

Le sel lavé est séché dans un dessiccateur, après évaporation de l'éther à l'air libre; il est conservé en resserre froide.

Dissoudre $1 \mathrm{gr} .09 \mathrm{du}$ sel lavé et séché dans $900 \mathrm{~cm}^{3}$ d'eau distillée, préalablement saturée de $\mathrm{CHCl}^{3}$. Ajouter $50 \mathrm{~cm}^{3}$ de solution tampon boratée, diluer jusqu'à un litre. Ajouter quelques gouttes de $\mathrm{CHCl}^{3}$. Conserver en resserre froide. Le $p H$ de ce substrat-tampon est d'environ 9,6 ; on le contrôle en utilisant la phtaléine de thymol comme indicateur.

\section{Standards des Colorations}

Préparer une solution de phénol dans de l'eau distillée standardisée à $0 \mathrm{em}^{3} \mathrm{I}$ par centimètre eube (30). Diluer le nombre requis de centimètres cubes de la solution jusqu'à $100 \mathrm{~cm}^{3}$, pour obtenir des solutions contenant $1 / 2,1,11 / 2,2,3,4,5,10,20,50$ et 100 parties de phénol par million. Pour préparer les standards de coloration, prendre $5 \mathrm{~cm}^{3}$ de chacune des solutions, $y$ additionner $1 / 4$ centimètre eube de la solution tampon boratée et deux gouttes de la solution $\mathrm{BQC}$; puis, bien fermer le tube. La coloration de ces préparations persiste inaltérée pendant plusieurs semaines, si on les conserve

(1) Ce sel libre de phénol peut être fourni par Ermer et AMend, New-York, ou par R. P. CARGILLE, New-York. 
en resserre froide et si, lors d'utilisation, on ne les expose pas à la lumière solaire directe.

Comme unité de bleu, est proposée la coloration produite par 0 mgr. 001 de phénol dans 5 centimètres cubes de solution. Sur cette base, le standard, qui contient $1 / 2$ partie de phénol pour un million, contiendrait 0 mgr. 0025 (2 gamma 5) de phénol par $5 \mathrm{~cm}^{3}$ ou 2 unités 5; le standard contenant 1 partie de phénol pour un million contiendrait $0 \mathrm{mgr} .005$ ( 5 gamma) par $5 \mathrm{~cm}^{3}$ ou 5 unités, etc.

\section{Méthode}

Chaque jour, les réactifs doivent être contrôlés. Si on obtient une coloration bleue, le substrat-tampon doit être rejeté.

$\mathrm{Au}$ moyen d'une pipette (une pipette pour chaque échantillon) on met $1 \mathrm{~cm}^{3}$ de lait dans une éprouvette de verre pyrex. La pipette doit être bouchée au moyen d'ouate pour prévenir l'introduction de salive dans l'échantillon. Ajouter $10 \mathrm{~cm}^{3} \mathrm{du}$ substrat-tampon et bien secouer, puis conserver pendant une heure à la température de $37^{\circ} 5$ C. Après l'incubation, placer les éprouvettes dans l'eau bouillante pendant 5 minutes, puis refroidir dans de l'eau glacée. Additionner $0 \mathrm{~cm}^{3} 1$ de la solution d'acétate basique de plomb, secouer et laisser reposer pendant une à deux minutes. Les protéines vont se coaguler et se précipiter. (Il est parfois nécessaire d'ajouter encore $0 \mathrm{~cm}^{3} 05$ d'acétate de plomb en plus pour obtenir une séparation complète de la protéine.) Filtrer et, à $5 \mathrm{~cm}^{3} \mathrm{du}$ filtrat limpide, ajouter d'abord $0 \mathrm{~cm}^{3} 25$ de la solution tampon boratée, ensuite 2 gouttes ou $0 \mathrm{~cm}^{3} 04$ de $\mathrm{BQC}$ et agiter légèrement.

La coloration bleue de la réaction de l'indophénol se développe en moins de cinq minutes. Après quinze minutes, jugées nécessaires pour le développement complet de la coloration, celle-ci est comparée avec les standards et évaluée en unités de phosphomonoestérase une unité étant la quantité d'enzyme qui, dans les conditions de l'expérience, produirait la coloration provoquée par 1 gamma de phénol.

\section{Précautions nécessaires lors de l'exécution de l'épreuve au Laboratoire}

A. Les échantillons de lait et de crème, surtout ceux qui ont été conservés en resserre froide, doivent être chauffés à la température du local.

B. Si on utilise une étuve plutôt qu'un bain-marie, il est bon de placer les éprouvettes pendant une à deux minutes dans un bainmarie à la température de $40^{\circ} \mathrm{C}$. environ, avant de les mettre dans l'étuve. 
C. Occasionnellement, après l'addition de l'acétate basique de plomb, le filtrat n'est pas limpide, ou il devient trouble après l'addition de un quart de centimètre cube de la solution tampon boratée. La centrifugation à une vitesse d'environ 800 tours à la minute clarifiera le sérum. Apparemment, le précipité ne prévient pas la formation d'indophénol.

D. Le papier-filtre Whatman $n^{\circ} 40$ fut utilisé au cours des recherches; mais, pour l'usage quotidien, n'importe quel papierfiltre de bonne qualité convient.

E. Toutes précautions doivent être prises pour éviter l'action de la phosphatase de la salive humaine.

\section{DONNÉES EXPÉRIMENTALES}

\section{Effet de la variation de la durée du chauffage et de la température}

Des échantillons de lait de mélange de troupeaux furent chauffés au laboratoire à la température de $143^{\circ} \mathrm{F}$. $\left(61^{\circ} 7 \mathrm{C}\right.$.) et maintenus à cette température pendant des périodes variant de 5 à 30 minutes. Ces échantillons furent examinés de même que des échantillons de lait prélevés, dans des conditions, autant que possible, identiques, dans quatre usines de pasteurisation, dans deux desquelles étaient utilisés comme chambreurs des réservoirs clos de petite dimension, tandis que dans les deux autres étaient utilisées des batteries de plusieurs chambreurs automatiques.

Il est possible que les durées de chauffage mentionnées pour les usines ne soient pas absolument exactes. Pour quelques échantillons, la période de maintien à la température de $143^{\circ} \mathrm{F}$. ( $61^{\circ} 7 \mathrm{C}$.) fut un peu plus longue à cause du temps nécessaire pour remplir le chambreur, avant la période de chambrage. Pour le lait cru de chacune des 34 vaches, la valeur de phosphomonoestérase fut de 500 unités et plus. Dans aucun cas, la coloration fournie par du lait adéquatement pasteurisé (au laboratoire ou à l'usine) n'indiqua plus de 2 unités 5 de phosphomonoestérase. L'expérience a démontré que cette donnée était un peu trop élevée; uniformément, on constate que l'activité des enzymes est en réalité moins élevée dans les échantillons adéquatement pasteurisés. Il ne peut être question de faculté de distinction entre du lait eru et du lait pasteurisé ; cette épreuve permet de déceler des variations de cinq minutes et plus de la durée du chauffage. La réaction du lait chauffé pendant 25 minutes est différente de celle du lait pasteurisé, celle du lait chauffé pendant 5 minutes est différente de celle du lait cru. Les données du tableau I montrent que la destruction de l'enzyme en question se fait plus efficacement dans un chambreur automatique de précision que dans 
TABLEAU I

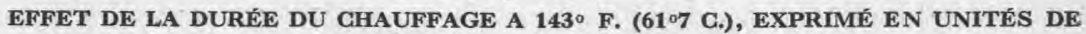
PHOSPHOMONOESTÉRASE PROPOSÉES

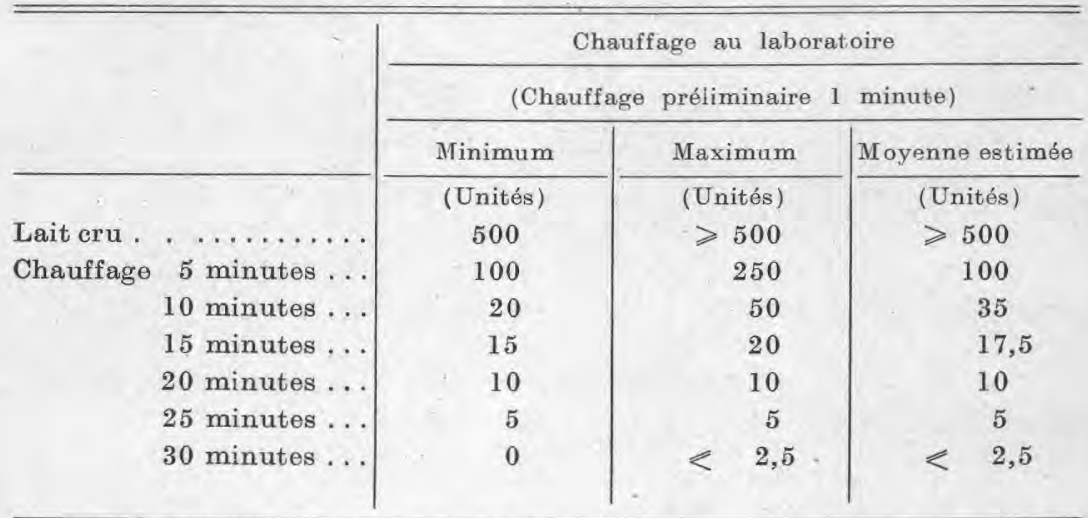

\begin{tabular}{|c|c|c|c|c|}
\hline & \multicolumn{4}{|c|}{ Echantillons d'usines de pasteurisation } \\
\hline & Usine A & Usine B & Usine C & Usine $\mathrm{D}$ \\
\hline & (Unités) & (Unités) & (Unités) & (Unités) \\
\hline Lait cru ....... & $\geqslant 500$ & $\geqslant 500$ & $\geqslant 500$ & $\geqslant 500$ \\
\hline Chauffage 5 minutes & 40 & 50 & 100 & $50-100$ \\
\hline 10 minutes & 20 & $20-25$ & 40 & 50 \\
\hline 20 minutes & 10 & 10 & 15 & 25 \\
\hline 25 minutes & $2,5-5$ & $2,5-5$ & $5-10$ & $5-10$ \\
\hline 30 minutes & 2,5 & 2,5 & 2,5 & 2,5 \\
\hline Lait en bouteilles ... & 2,5 & 2,5 & $<2,5$ & 2,5 \\
\hline
\end{tabular}

Usine A. - Chauffage préliminaire de 60 secondes environ dans un appareil I-T. Plusieurs chambreurs automatiques de précision.

Usine B. - Chauffage préliminaire de 60 secondes environ dans un appareil I-T. Il fallut six minutes et demie pour le remplissage d'un chambreur. Six chambreurs Cherry Burrell Spray. Il fallut trois minutes pour la vidange d'un chambreur. Réfrigération du lait à $50^{\circ} \mathrm{F}$. $\left(10^{\circ} \mathrm{C}\right.$.) en 30 secondes.

Usine C. - Chambrage dans petit réservoir elos. Chauffage préliminaire jusqu'à $143^{\circ} \mathrm{F}$. $\left(61^{\circ} 7 \mathrm{C}\right.$.) en 15 minutes. Réfrigération au moyen d'un réfrigérateur découvert.

Usine D. - Chambrage dans petit réservoir clos avec agitateur en mouvement continu. La durée du chauffage préliminaire, dans le réservoir, était de 15 minutes. Réfrigération dans le réservoir en cinq minutes.

un réservoir chambreur d'un type plus ancien et que le chauffage préliminaire de courte durée était plus efficace pour la destruction de l'enzyme que le chauffage préliminaire de plus longue durée. 
On ne peut cependant perdre de vue que ces indications ne sont fournies que par un nombre très limité de données.

Des échantillons de lait de mélange d'un troupeau furent chauffés au laboratoire, pendant 30 minutes, à des températures différentes. Les résultats sont résumés dans le tableau II.

TABLEAU II

EFFET DE LA VARIATION DE LA TEMPÉRATURE D'UN CHAUFFAGE D'UNE DURÉE DE TRENTE MINUTES, EXPRIMÉ EN UNITÉS DE PHOSPHOMONOESTÉRASE PROPOSEEES

\begin{tabular}{c|c}
\hline \hline Température & Valeur moyenne (Unités) \\
\hline & \\
$138^{\circ}$ F. $\left(58^{\circ} 9\right.$ C.) & $250-50$ \\
$140^{\circ}, 5$ F. $\left(60^{\circ} 28\right.$ C.) & 100 \\
$142^{\circ}$ F. $\left(61^{\circ} 1\right.$ C.) & 10 \\
$143^{\circ}$ F. $\left(61^{\circ} 7\right.$ C.) & 2,5 \\
\hline
\end{tabular}

L'effet de l'addition de lait cru, dans des proportions différentes, à du lait adéquatement pasteurisé est montré dans le tableau III. On voit que l'addition de $0,5 \%$ de lait cru à du lait adéquatement pasteurisé peut être décelée sans difficulté.

Des observations au cours des six derniers mois sur du lait pasteurisé au laboratoire et sur du lait provenant d'usines de pasteurisation, ont démontré que chaque fois que la donnée obtenue au moyen de cette méthode était supérieure à 2 unités 5 , le chauffage avait été insuffisant ou la pasteurisation avait été défectueuse et que l'élévation de la valeur obtenue était en proportion directe avec la défectuosité de la pasteurisation. Il n'est cependant pas possible, en se basant sur les données obtenues par cette épreuve, de déterminer, sans enquête ultérieure, si le défaut est dû à la durée du chauffage, ou à une température trop peu élevée, ou à l'addition au lait pasteurisé (à cause d'un défaut de l'appareil) de lait cru ou insuffisamment chauffé, ou à un mélange, au lait, de mousse insuffisamment chauffée, ou à une association de ces facteurs. L'expérience a encore démontré qu'il n'est pas nécessaire de faire chaque observation en double. Si on veut avoir toute garantie de l'absence de phénol ou de produits similaires, qui pourraient donner une réaction avec BQC, on peut substituer une solution aqueuse (ajustée au $p \mathrm{H} 9,6$ ) au substrat-tampon, en $\mathrm{y}$ appliquant la méthode décrite ou la méthode de "campagne" rapide, qui sera décrite plus loin, dans ce mémoire. Il peut être intéressant de signaler que même lorsque des échantillons de lait dit "pasteurisé " du commerce fournirent un index de 500 unités 
après incubation avec le substrat, l'épreuve témoin indiqua l'absence de phénol.

\section{Effet des conditions de conservation}

On a constaté $[14,20]$ que lorsque du lait s'est acidifié ou s'est putréfié, on peut obtenir une coloration plus prononcée avec l'épreuve de Kay et Graham, grâce à l'action bactérienne ou à la décomposition des protéines, ce qui peut libérer du phénol ou des produits similaires au phénol. Dans ces conditions, on pourrait considérer du lait pasteurisé efficacement comme un produit mal pasteurisé. Certains de ces produits de la décomposition, tels la tyrosine, le tryptophane, donneront une coloration bleue avec le réactif de Folin et Ciocalteu [25]. Un examen de la formule de structure de ces produits et de produits apparentés démontre qu'il y a déjà eu substitution de place du groupe $\mathrm{OH}$, ou que le composé ne contient pas de groupe OH. Dans ces cas, la théorie indiquerait qu'il ne peut y avoir de réaction avec $\mathrm{BQC}$ pour donner lieu à la formation d'indophénol.

Quarante-deux échantillons de lait, provenant de différentes usines (après chauffage à $143^{\circ} \mathrm{F}$. $\left(61^{\circ} 7 \mathrm{C}\right.$.) pendant des périodes différentes) et 22 échantillons traités au laboratoire (durées différentes, températures différentes de chauffage, mélanges de lait pasteurisé et de lait eru), furent conservés à des températures de réfrigération et l'on pratiqua, de temps en temps, sur ces divers échantillons, l'épreuve de la phosphomonoestérase. Les tableaux III, IV, V donnent les résultats obtenus.

TABLEAU III

GONSERVATION DE LAIT PASTEURISE ADDITIONNÉ DE LAIT CRU

\begin{tabular}{|c|c|c|c|}
\hline \multirow{2}{*}{$\begin{array}{c}\text { Pourcentage de lait eru } \\
\text { additionné }\end{array}$} & \multicolumn{3}{|c|}{ Unités de phosphomonoestérase } \\
\hline & 1 jour & 7 jours & 14 jours \\
\hline 8 & & & \\
\hline $0,0 \ldots \ldots \ldots \ldots \ldots \ldots \ldots$ & $<2,5$ & $<2,5$ & $<2,5$ \\
\hline $0,2 \ldots \ldots \ldots \ldots \ldots \ldots \ldots$ & $<5$ & $<5$ & $<5$ \\
\hline $0,5 \ldots \ldots \ldots \ldots \ldots \ldots \ldots \ldots$ & 5 & 5 & 5 \\
\hline $1,0 \ldots \ldots \ldots \ldots \ldots \ldots \ldots \ldots$ & 10 & $\geqslant 10$ & $<10$ \\
\hline $2,0 \ldots \ldots \ldots \ldots \ldots \ldots \ldots$ & $15-20$ & $\geqslant 10$ & $\geqslant 10$ \\
\hline $5,0 \ldots \ldots \ldots \ldots \ldots \ldots \ldots$ & $20-50$ & & t \\
\hline $10,0 \ldots \ldots \ldots \ldots \ldots \ldots \ldots \ldots$ & 100 & & \\
\hline
\end{tabular}


TABLEAU IV

EFFET DE LA CONSERVATION DE LAIT PASTEURISE INDUSTRIEL SUR L'ÉPREUVE

\begin{tabular}{|c|c|c|c|c|c|c|c|c|}
\hline \multirow{2}{*}{$\begin{array}{l}\text { Indications } \\
\text { des } \\
\text { inspecteurs }\end{array}$} & \multicolumn{7}{|c|}{ Nombre de jours de conservation } & \multirow{2}{*}{$\begin{array}{l}\text { Titrage au } \\
p \mathrm{H} 6,6 \text { avec } \\
\mathrm{Na}^{2} \mathrm{CO}^{3} \text { avant } \\
\text { la prise de } \\
\text { l'échantillon } \\
\text { (50 jours) }\end{array}$} \\
\hline & 1 & 6 & 10 & 20 & 27 & 35 & 50 & \\
\hline $\mathrm{Cru} \ldots \ldots$. & 500 & 500 & 500 & $\geqslant 100$ & 15 & 15 & 15 & 500 \\
\hline 5 minutes. & 100 & 100 & 20 & 10 & 15 & $5 \cdot 10$ & & \\
\hline 10 minutes. & 25 & 20 & 10 & 5 & 10 & 510 & & \\
\hline 15 minutes. & 10 & 10 & 5 & 2,5 & 5 & 5.10 & 2,5 & 5 \\
\hline 20 minutes & 5 & $>2,5$ & $>2,5$ & $<5$ & $<5$ & $<5$ & & \\
\hline 25 minutes. & 2,5 & 2,5 & $<2,5$ & $<2,5$ & $<2,5$ & $<2,5$ & & \\
\hline 30 minutes. & $<2,5$ & $<2,5$ & $<2,5$ & $<2,5$ & $<2,5$ & $<2,5$ & $<2,5$ & $<2,5$ \\
\hline
\end{tabular}

TABLEAU V

EFFET DE LA GONSERVATION SUR DES ÉGHANTILLONS GHAUFFES A MOINS DE $143^{\circ}$ F. (617 G.) PENDANT 30 MINUTES, EXPRIMÉ EN UNITÉS PROPOSÉES

\begin{tabular}{|c|c|c|c|c|c|c|}
\hline \multirow{2}{*}{ Température } & \multicolumn{5}{|c|}{ Jours de conservation } & \multirow{2}{*}{$\begin{array}{l}\text { Titrage avec } \\
\mathrm{Na}^{2} \mathrm{CO}^{3} \text { avant } \\
\text { la prise de } \\
\text { l'échantillon } \\
\text { après } 27 \text { jours }\end{array}$} \\
\hline & 1 & 3 & 6 & 10 & 27 & \\
\hline $138^{\circ}$ F. $\left(58^{\circ} 9\right.$ C. $)$ & 500 & 500 & 100 & 100 & 10 & $50-100$ \\
\hline $140^{\circ} 5$ F. $\left(60^{\circ} 3\right.$ C. $)$ & 100 & $50-100$ & 50 & 15 & $5-10$ & 20 \\
\hline $142^{\circ} \mathrm{F} .\left(61^{\circ} 1 \mathrm{C}.\right)$ & 10 & $5-10$ & 10 & 5 & 2,5 & $2,5-5$ \\
\hline $143^{\circ} \mathrm{F} .(6107$ C. $)$ & 2,5 & 2,5 & 2,5 & 2,5 & 2,5 & 2,5 \\
\hline
\end{tabular}

Les laits dont la pasteurisation avait été inadéquate fournirent des données confirmant cette particularité, après une longue période de conservation. La baisse apparente des unités de phosphomonoestérase peut avoir été provoquée en même temps par la hausse du degré d'acidité et par le fait que, dans ces conditions, le $p H \mathrm{H}$ du substrat-tampon ne put être maintenu à l'optimum indiqué. Il y eut encore de la difficulté pour obtenir un échantillon homogène, à cause de l'altération des laits. Quelques-uns des laits furent ajustés à un $p \mathrm{H}$ d'environ 6,6 , au moyen de $\mathrm{Na}^{2} \mathrm{CO}^{3}$ avant la détermination des unités de phosphomonoestérase; les données, ainsi obtenues, sont très intéressantes. 
TABLEAU VI

RÉSULTATS DE LAGIDIFICATION AU GOURS DE LA GONSERVATION, EXPRIMÉS EN UNITÉS PROPOSEEES

\begin{tabular}{|c|c|c|c|c|c|c|}
\hline & \multirow{2}{*}{$\begin{array}{l}\text { No de } \\
\text { l'échan- } \\
\text { tillon }\end{array}$} & \multicolumn{5}{|c|}{ Jours de conservation } \\
\hline & & 1 & 7 & 10 & 20 & 50 \\
\hline \multirow{4}{*}{ Lait cru } & 1 & 500 & 500 & & 500 & 500 \\
\hline & 2 & 500 & & & 500 & 500 \\
\hline & 3 & 500 & & 500 & & 500 \\
\hline & 4 & 500 & 500 & 500 & $100+$ & \\
\hline \multirow{5}{*}{$\begin{array}{l}\text { Chauffage } \\
\text { préliminaire }\end{array}$} & $5 \mathrm{~A}$ & 500 & & & & 500 \\
\hline & $6 B$ & 500 & & & 500 & 100 \\
\hline & $7 \mathrm{C}$ & 500 & & & & 500 \\
\hline & $8 \mathrm{D}$ & 500 & & & & \\
\hline & $9 \mathrm{~A}$ & 15 & 15 & 15 & & 5 \\
\hline \multirow{6}{*}{$\begin{array}{l}10 \text { minutes à } \\
143^{\circ} \mathrm{F} .\left(61^{\circ} 7 \mathrm{C} .\right)\end{array}$} & $10 \mathrm{~A}$ & 40 & 40 & 40 & & 20 \\
\hline & $11 \mathrm{~A}$ & 25 & 20 & 10 & 5 & 5 \\
\hline & $12 \mathrm{C}$ & 50 & 50 & 50 & & \\
\hline & $13 \mathrm{D}$ & 20 & 20 & 20 & & 10 \\
\hline & $14 \mathrm{~A}$ & 20 & 25 & 20 & 15 & \\
\hline & $15 \mathrm{~A}$ & 25 & 25 & 20 & & \\
\hline \multirow{7}{*}{$\begin{array}{c}20 \text { minutes à } \ldots . . . \\
143^{\circ} \mathrm{F} .\left(61^{\circ} \mathrm{C} .\right)\end{array}$} & $16 \mathrm{~B}$ & 5 & & & & 2,5 \\
\hline & 17B & 10 & 10 & $<10$ & & $<5$ \\
\hline & $18 \mathrm{~B}$ & 5 & 5 & 2,5 & 2,5 & \multirow{3}{*}{2,5} \\
\hline & $19 \mathrm{C}$ & 25 & 20 & & & \\
\hline & $20 \mathrm{D}$ & 10 & 10 & 5 & . & \\
\hline & $21 \mathrm{~A}$ & 10 & 10 & 10 & 5 & 2,5 \\
\hline & $22 \mathrm{~A}$ & 10 & 10 & 10 & 5 & \\
\hline
\end{tabular}

A : Chauffage préliminaire au laboratoire, 1 minute.

B : Chauffage préliminaire industriel, 1 minute et $1 / 2$.

C : Chauffage préliminaire industriel, 15 minutes.

D : Chauffage préliminaire industriel, 15 minutes avec agitation dans le réservoir.

\section{Application de l'épreuve aux produits lactés}

L'épreuve fut appliquée à des échantillons de crème crue et de crème pasteurisée, provenant d'usines fournissant toutes garanties ; il ne fallut aucune modification de technique. Pour les crèmes crues, la valeur fut de 500 unités et plus; pour les crèmes pasteurisées, elle fut de 2,5 unités (dans deux cas, elle fut de 5 unités).

Il ne fallut aucune modification de la technique pour le lait au 
chocolat. Des recherches, encore en cours, indiquent que l'épreuve pourra être appliquée au beurre et à d'autres produits lactés, avec de légères modifications de la méthode, mais à cause du nombre très limité d'observations, les données obtenues ne sont pas encore publiées.

\section{Pasteurisation de courte durée et à température élevée}

Des échantillons de lait pasteurisé dans un appareil de pasteurisation à température élevée, type York, fournirent la même réaction à l'épreuve que le lait ayant subi la pasteurisation ordinaire. Cet appareil "était censé chauffer le lait à $160^{\circ} \mathrm{F}$. $\left(71^{\circ} 1 \mathrm{C}\right.$.) pendant 15 secondes. La durée réelle du chauffage, déterminée en faisant passer de l'eau dans l'appareil, était de 17 à 18 secondes.

\section{Application de l'épreuve proposée, à du lait et de la crème pasteurisée du commerce}

Il n'est pas possible de publier des tableaux des données obtenues aveo les nombreux échantillons examinés, mais le tableau VII montre quelques données représentatives eoncernant du lait et de la crème pasteurisés du commerce. Parmi les échantillons exami-

TABLEAU VII

QUELQUES RESULTATS OBTENUS AVEG DES EGHANTHLONS DE LATT ET DE GRÈME DU GOMMERGE, DESIGNÉS COMME PASTEURISÉS

\begin{tabular}{|c|c|c|}
\hline $\begin{array}{l}\text { Nature des éehan- } \\
\text { tillons }\end{array}$ & $\begin{array}{c}\text { Unités } \\
\text { proposées }\end{array}$ & Observations \\
\hline Crème queleonque & 100 & Pasteurisation inadéquate. \\
\hline Lait officiel ..... & 20 & $\begin{array}{l}\text { Pompes mal réglées, mélange lait chauffé et } \\
\text { cru, durée du chauffage trop brève. }\end{array}$ \\
\hline Crème officielle & 500 & $\begin{array}{l}\text { Les registres de l'usine indiquèrent la pasteu- } \\
\text { risation de quantités supérieures de } 20 \% \text { au } \\
\text { débit possible des appareils. }\end{array}$ \\
\hline Lait officiel .... & 15 & Durée de chambrage de 20 minutes. \\
\hline Lait officiel ..... & 15 & Thermo mètre inexact de $2^{\circ} \mathrm{F}$. \\
\hline Crème officielle ... & 10 & Crème standardisée après la pasteurisation au \\
\hline Crème officielle ... & 25 & $\begin{array}{l}\text { moyen de lait cru ou imparfaitement pas- } \\
\text { teurisé. }\end{array}$ \\
\hline Lait officiel & 7,5 & Mousse en quantité dans le chambreur. \\
\hline Crème officielle ... & 7,5 & $\begin{array}{l}\text { Arrêt des agitateurs au cours de la pasteurisa- } \\
\text { tion. }\end{array}$ \\
\hline Lait quelconque & 15 & Température inférieure à $142^{\circ} \mathrm{F} .\left(61^{\circ} \mathrm{l}\right.$ C. $)$ \\
\hline
\end{tabular}

nés se trouvent des échantillons quelconques, prélevés dans le commerce, et des échantillons officiels, prélevés à l'usine. Les teneurs 
en matière grasse et en constituants solides des échantillons quelconques furent prélevés d'abord, ensuite leur fut appliquée l'épreuve de la phosphomonoestérase. Lorsque le résultat obtenu permettait de supposer une pasteurisation inadéquate, un échantillon officiel était prélevé, le jour même, à l'usine et, à cet échantillon, l'épreuve de la phosphomonoestérase était seule appliquée. Fréquemment, dans ces cas, des inspecteurs, munis de l'outillage de "campagne" rapide, visitèrent l'usine, le soir même, au cours de la pasteurisation, et contrôlèrent les travaux. Par ce système, des défauts de technique et des défectuosités des appareils furent découverts et signalés au personnel de l'usine.

On a affirmé que le refroidissement lent du lait pasteurisé par le maintien pendant 20 à 40 minutes à une température déterminée avait comme résultat la réactivation partielle de la phosphomonoestérase et que, lors de réfrigération rapide, la destruction de la phosphatase était plus complète [33]. Jusqu'ici l'Auteur n'a pas eu confirmation de cette affirmation, au cours de ses expériences, et la question ne constitue pas un problème puisque la pasteurisation adéquate exige une réfrigération rapide du lait.

Folley et KAy ont démontré [31] que la teneur en phosphomonoestérase du lait de vache varie au cours de la lactation, qu'elle est à son maximum 180 jours après la parturition. La teneur en enzymes du lait peut varier d'après la vache, mais ce fait a peu d'importance pour le lait de commerce, qui est généralement du lait de mélange ou du lait d'un troupeau. Ces auteurs ont encore démontré [29] que la teneur en enzymes du lait cru présente un rapport avec ses solides autres que la matière grasse.

Actuellement, des études sont en cours, en vue de rechercher si une incubation plus prolongée n'augmenterait pas la sensibilité de l'épreuve ou accentuerait sa valeur comme moyen de diagnostic. Cependant, en présence du fait que la rapidité de l'hydrolyse diminue avec la prolongation de la durée de la réaction et que l'activité d'une solution de phosphatase n'est pas proportionnelle à sa concentration, une durée d'incubation brève, dans laquelle l'activité enzymatique est plus proche de la vélocité initiale réelle de l'hydrolyse, paraît mieux convenir. Des recherehes sont faites concernant l'effet catalytique des ions de magnésium [32] sur la sensibilité.

\section{Epreuve rapide de campagne}

A cause de sa simplicité, la méthode, qui vient d'être décrite comme méthode de laboratoire, peut être adaptée de façon à pouvoir être utilisée comme méthode de campagne ; cette adaptation est facilitée par le fait que la présence de la matière grasse et des protéines du lait ne cache pas la coloration bleue de la réaction 
de l'indophénol. Pour cette méthode de eampagne simplifiée, on trouve le matériel dans une trousse (1) de dimensions réduites (fournie par R. P. CARGILlE).

A $5 \mathrm{~cm}^{3}$ de substrat-tampon on ajoute $0 \mathrm{~cm}^{3} 5$ de l'échantillon de lait ou de crème. On ferme au moyen d'un bouchon, et on agite vigoureusement. Pour l'incubation, on place l'éprouvette dans une poche ou on la plonge pendant 5 à 10 minutes dans un bain-marie à $100^{\circ} \mathrm{F}$. $\left(37^{\circ} 8 \mathrm{C}\right.$.), puis on ajoute 6 gouttes de $\mathrm{BQC}$, on remet le bouchon et on agite. On observe la couleur après 5 minutes. L'apparition d'une coloration bleue, quelle que soit son intensité, indique une pasteurisation défectueuse.

On peut trouver dans le commerce des comprimés pouvant servir à la préparation des deux solutions nécessaires.

\section{Application de l'épreuve de campagne}

TABLEAU VIII

\begin{tabular}{|c|c|}
\hline Echantillon & $\begin{array}{l}\text { Intensité de la } \\
\text { eoloration bleue }\end{array}$ \\
\hline$\ldots \ldots \ldots \ldots \ldots \ldots$ & +++ \\
\hline Lait à chauffage préliminaire de $120^{\circ} \mathrm{F}$. $\left(48^{\circ} 9 \mathrm{C}\right.$. $) \ldots \ldots \ldots$ & $++t+$ \\
\hline Lait à chauffage préliminaire de $143^{\circ} \mathrm{F} .\left(61^{\circ} 7\right.$ C. $) \ldots \ldots \ldots$ & +++ \\
\hline Lait chauffé pendant 10 minutes à $143^{\circ} \mathrm{F} .\left(61^{\circ} 7\right.$ C. $) \ldots \ldots$ & ++ \\
\hline Lait chauffé pendant 15 minutes à $143^{\circ} \mathrm{F} .\left(61^{\circ} 7 \mathrm{C}.\right) \ldots \ldots$ & + \\
\hline Lait chauffé pendant 20 minutes à $143^{\circ} \mathrm{F} .\left(61^{\circ} 7 \mathrm{C}.\right) \ldots \ldots$ & \pm \\
\hline Lait chauffé pendant 30 minutes à $143^{\circ} \mathrm{F} .\left(61^{\circ} 7 \mathrm{C}.\right) \ldots \ldots$ & - \\
\hline $5 \%$ de lait cru + du lait pasteurisé $\ldots \ldots \ldots \ldots \ldots \ldots$ & ++ \\
\hline $2 \%$ de lạit cru + du lait pasteurisé $\ldots \ldots \ldots \ldots \ldots \ldots$ & + \\
\hline $1 \%$ de lait eru + du lait pasteurisé $\ldots \ldots \ldots \ldots \ldots \ldots$ & \pm \\
\hline 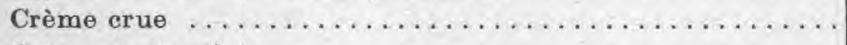 & $+t+t$ \\
\hline 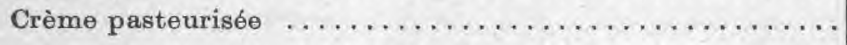 & 一. \\
\hline
\end{tabular}

La coloration bleue de l'indophénol se développe après moins de 5 minutes et devient progressivement plus intense. La crème adéquatement pasteurisée donne une coloration variant du gris au blane, le lait adéquatement pasteurisé une coloration variant du gris au brun. Il a été démontré que chaque fois que l'épreuve de laboratoire indique 10 unités ou plus, l'épreuve de campagne donne une coloration bleue. Dans certains cas, l'épreuve de eampagne donna une réaction positive avec de la crème, lorsque l'épreuve de laboratoire n'indiqua que 7 unités 5 de couleur. L'épreuve de campagne a même décelé le mélange de mousse insuffisamment chauffée

(1) Cette trousse contient, outre le réactif, des éprouvettes à fond plat jaugés à $5 \mathrm{~cm}^{3}$ et $5 \mathrm{~cm}^{3} 5$. 
au produit pasteurisé. Lorsque les données fournies par l'épreuve de campagne sont douteuses, l'épreuve de laboratoire doit être appliquée.

Le cas suivant démontre l'utilité des deux épreuves. Il fut constaté que de la crème de l'usine $\mathrm{X}$, lors de l'application de l'épreuve de laboratoire, donnait un nombre élevé d'unités. Cette usine ne faisait que la mise en bouteilles de crème "pasteurisée " en vrac, reçue d'environ 20 usines rurales. Des inspecteurs découvrirent la cause en utilisant l'épreuve de campagne. Un lot de crème fournit une réaction très prononcée de la phosphomonoestérase, ce qui amena l'examen et la saisie d'un lot de erème très important. L'épreuve de laboratoire démontra que cette grande quantité de crème ne présentait pas une réaction uniforme ; elle put être répartie en trois catégories selon la réaction : 500 unités, 100 unités, 20 unités. Un inspecteur de la Division de contrôle des Laiteries rurales (Country Milk Division), lors de la visite de l'usine, constata des irrégularités du contrôle de la température dans un chambreur (le tracé des températures indiquait $143^{\circ} \mathrm{F} .\left(61^{\circ} 7 \mathrm{C}\right.$ ), mais le thermomètre avait glissé et sa cuvette reposait sur le fond du réservoir, fait qui avait passé inaperȩu, et la température réelle n'était que de $139^{\circ}$ F. $\left(59^{\circ} 9\right.$ C. $)$. Cette circonstance, avec, en plus, un défaut d'agitation au cours du chambrage, et la constatation, faite ultérieurement, que cette crème " pasteurisée " était encore standardisée, s'il était nécessaire, au moyen de lait, qui, également, avait été l'objet d'une pasteurisation défectueuse, constituait probablement la cause des variations du nombre d'unités de phosphomonoestérase constaté. Ces irrégularités furent supprimées, et, depuis lors, cette crème fournit régulièrement un indice de moins de 2 unités 5 .

\section{Corrélation entre les teneurs bactériennes et les unités de phosphomonoestérase}

L'activité de la phosphomonoestérase de plusieurs milliers d'échantillons de lait et de crème pasteurisés du commerce, dont la teneur bactérienne avait été contrôlée, fut déterminée.

Comme il est évident que d'autres facteurs, qu'une pasteurisation adéquate, peuvent avoir de l'influence sur la teneur bactérienne, ces données ne sont pas publiées. De nombreux échantillons à teneur bactérienne élevée présentèrent un indice de phosphomonoestérase de 2 unités 5 . Cette teneur bactérienne élevée peut être due à une teneur bactérienne élevée initiale du lait cru, à des bactéries thermorésistantes, à l'âge du lait, à une infection ultérieure à la pasteurisation. On ne peut cependant perdre de vue qu'un lait, à teneur bactérienne peu élevée, n'est pas toujours un lait sans danger.

L'indice de phosphomonoestérase d'un échantillon de lait ne 
fournit pas d'indication sur une contamination du produit ultérieure à la pasteurisation.

\section{Discussion de la valeur pratique de l'épreuve}

$A u$ cours du premier mois d'application de cette épreuve, au cours duquel furent considérés anormaux (c'est-à-dire à réaction positive) seulement les laits et les crèmes dont l'indice de phosphomonoestérase était supérieur à 5 unités et fut appliquée l'épreuve de campagne simplifiée dans les usines et les laiteries suspectes; 23 livres 977 (10.874 kg. 53) de lait et de crème furent qualifiées inadéquatement pasteurisées et exclues du marché de la ville de New-York.

Le travail fait jusqu'ici démontre que la pasteurisation défectueuse est involontaire ou préméditée.

La pasteurisation involontaire peut être due à :

a) Un outillage mauvais ou inadéquat.

b) La négligence dans l'utilisation d'un outillage adéquat.

c) Un défaut de contrôle du fonctionnement des appareils.

d) Un défaut de contrôle de l'exactitude des thermomètres de l'usine, par eomparaison avec un thermomètre du Bureau des Standards.

e) Un défaut d'élimination d'une formation excessive de mousse.

f) Une fluctuation de la température à cause du défaut de contrôle automatique.

La pasteurisation défectueuse préméditée peut être due à :

a) Une baisse de la température à $140^{\circ} \mathrm{F}$. $\left(60^{\circ} \mathrm{C}\right.$.) ou moins, provoquée intentionnellement en vue d'obtenir une couche de crème apparemment plus épaisse.

b) L'arrêt des agitateurs dans le même but.

c) Une réduction de la période de chambrage, dans le but de maintenir le débit régulier de l'usine, lors d'une mise hors de service temporaire d'une partie de l'outillage.

d) Le relâchement du contrôle des opérations au cours de la pasteurisation du dernier lot de lait de la journée de travail.

e) La suppression intentionnelle de la période de chambrage.

f) L'addition de lait cru à du lait pasteurisé dans le but d'obtenir le volume de lait désiré, ou la standardisation de crème au moyen de lait cru.

g) Le défaut d'inhibition de la formation de mousse.

\section{Résumé}

Après une revue de la littérature, une méthode rapide de contrôle de la pasteurisation de lait, de crème et d'autres produits de laiterie 
est décrite. L'hydrolyse enzymatique d'un substrat est déterminée, quantitativement, par l'addition de 2,6 dibromoquinonechloroimide, donnant lieu à des colorations bleues d'indophénol d'intensité variable, lorsque la pasteurisation est défectueuse. L'épreuve est exécutée en moins d'une heure et demie et indique des défauts peu évidents de la technique de la pasteurisation, tels que :

1. Une température inférieure de $1^{\circ} \mathrm{F}$. $\left(0^{\circ} 55 \mathrm{C}\right.$.) à celle de $143^{\circ} \mathrm{F}$. $\left(61^{\circ} 7\right.$ C. $)$ exigée par le Code sanitaire de la ville de New-York.

2. Un chauffage de 25 minutes au lieu de la période de 30 minutes à $143^{\circ} \mathrm{F}$. $\left(61^{\circ} 7\right.$ C.) qui est exigée.

3. L'addition de $0,5 \%$ de lait cru à de la crème ou du lait adéquatement pasteurisés.

La méthode n'exige pas d'appareils spéciaux; elle est peu onéreuse et peut être appliquée à l'usine en vue du contrôle de l'efficacité de la pasteurisation. Une méthode d'expression des résultats en unités de phosphomonoestérase proposées est préconisée.

Une épreuve de campagne simple, dont l'application exige environ dix minutes, qui peut être utilisée par des inspecteurs et présente une valeur réelle comme moyen de diagnostic est déerite.

\section{Remerciements}

L'Auteur remercie, pour leur collaboration, Harry W. RAYBIN, le chimiste en fonetion Reginald MrLLer et d'autres membres du personnel du Laboratoire Chimique, les Chefs et Inspecteurs de la Division de Laiterie du Bureau des Aliments et Médicaments du Service d'Hygiène de la ville de New-York, le chimiste Morris PaLeY du personnel de la Bibliothèque WPA.

\section{BIBLIOGRAPHIE}

[1] Associates of Lore A. Rogers. Fundamentals of Dairy Science, édition 1928 , p. 364.

[2] W. D. Frost et G. D. Moore. The cellular test for pasteurized milk under practical conditions. Journal Dairy Science, 2, 1919, 189.

[3] S. Orla-Jensen. Proben zur Feststellung des Erhitzungsgrade der Milch. Z. Untersuch. Lebensm., 63, 1932, 300.

[4] M. F. Bengen. Nachweis de Dauerpasteurisierung. Z. Untersuch. Lebensm., 66, 1933, 129.

[5] D. LANDANy. A chemical method for identication of pasteurized, boiled and sterilized milk. Bull. Soc. Chim. Yougoslav., 2, 1931, 57.

[6] G, Z Milch. Mitt. Lebensmitteluntersuch. u. Hygiene, 25, 1934, 87.

[7] F. G. Kонn et E. Klemm. Sur le diagnostic du lait pasteurisé à basse température par l'anneau de Schern-Gorli. Le Lait, 12, 1932, 19. 
[8] S. Rothenfusser. Uber den Nachweis der Dauerpasteurisierung. Z. Untersuch. Lebensm., 60, $1930,94$.

[9] P. G. Heinemann. Milk. W. B. Saunders Co., p. 237.

[10] E. VAN Kostler. Swiss Provisions book, $3^{\text {e }}$ édition, p. 14.

[11] F. SCHARDINGer. Unterscheidung gekochter und ungekochter Milch mittels Methylenblau. Z. Nahr. Genussm., 5, 1902, 1113.

[12] B. S. GoULD. The detection of inefficient pasteurized milk based on a modification of the new Rothenfusser test. Journal Dairy Science, $15,1932,230$.

[13] H. W. LeAHy. A practical test of pasteurization. 23rd Annual Report International Association of Dairy and Milk Inspectors, 1934.

[14] H. D. KAY et W. R. (Jr) GRAHAM. The phosphatase test for pasteurized milk. Journal Dairy Res., 6, 1935, 191.

[15] M. F. Bengen et E. Bohm. Nachweis der Pasteurisierung von Milch. Z. Untersuch. Lebensm, 67, 1934, 395. - Ist die Amylase Probe zum alleinigen Nachweis genügender Dauererhitzung geeignet. Ibid, 69, $1935,146$.

[16] P. Weinstein. Nachweis des Erhitzungsgrade der Mileh. Ibid, 56, 1928, 457. - Nachweis in der Milch. Ibid, 68, 1934, 73.

[17] C. Lind. Les enzymes du lait et leur utilisation pour appréejer le chauffage du lait. Le Lait, 7, 1927, 935 .

[18] K. Eble et H. Pferffer. Zur Erkennung Dauererhitzter Mileh. Z. Untersuch. Lebensm., 60, 1930, 311.

[19] A. Schlözmer. Die amylase de Kuhmilch als $\alpha$-amylase. Ibid, 71, 1936,311 .

[20] F. W. Giloreas et W. S. Davis. An investigation of the amylase and phosphatase tests as an indication of pasteurization. Annual Proceedings of the Internatioanl Association of Milk Sanitarians, 1936 , p. 15.

[21] Methods for detection of pasteurized milk. U. S. Department of Agriculture, Bureau of Chem., bull. $\mathrm{n}^{\circ} 162,1913$.

[22] G. A. Richardson et C. L. Harkinson. Amylase in Cow Milk. Journal Dairy Science, 19, 1936, 761.

[23] G. G. Freeman et R. H. Hopkins. Mechanism of the degradation of starch by amylases. Bioch. Journ., 3o, 1936, 442.

[24] E. B. Anderson, Z. Herschdorfer et F. K. NeAve. The Enzymes of Milk. Analyst., 62, 1937, 86.

[25] O. Folin et V. Ciocaltev. On tyrosine and tryptophane determinations in proteins. Journal Biol. Chem., 73, 1927, 627.

[26] H. D. Gibss. Phenol tests. III. The indophenol test. Journal Biol. Chem., 72, 1927, 649 .

[27] E. J. King et A. R. Armstrong. A convenient method for determining serum and bile phosphatase activity. Canadian Med. Assoc. Journ., 31, $1934,376$.

[28] S. J. Foldey et H. D. KAY. The alkaline phosphomonoestérase of the mammary gland, Biochem. Journ., 29, 1935, 1837.

[29] S. J. Folley et H. D. KAY. Enzymes of the mammary gland. Journal Dairy Res., vol. VIII, 1937, 111 (Biennial Review of the progress of Dairy Science). 
[30] U. S. Pharmacopoeia, $11^{\text {e }}$ édition, p. 285.

[31] J. S. Follex et H. D. KAY. Variation in the phosphomonoesterase content of milk of the cow in relation to the progress of lactation. Enzymologia, 1, 1936, 48 .

[32] S. J. Folley et H. D. KAy. The phosphatases. Ergebnisse fur Enzymfursch., 5, 1936, 159.

[33] S. Herschdorfer. Inactivation of phosphate. Enzymologia, 1, 1936,96.

\section{BIBLIOGRAPHIE ANALYTIQUE}

\section{JOURNAUX, REVUES, SOGIÉTÉS SAVANTES ECONOMIE LAITIERE}

KAY (H. D.). - Augmentation de la production du lait. The Scottish Journal of Agriculture, vol. XX, nº 1, janvier 1937.

K. examine le problème de l'augmentation de la production du lait en Grande-Bretagne. La consommation journalière de lait par tête d'habitant y a été, en 1934, de 01.227 ; en 1935-1936 de 01.250 , alors qu'aux Etats-Unis, elle varie actuellement entre 0 1. 238 (Saint-Louis) et 0 1. 443 (Boston). Cette consommation s'élève toutefois en Grande-Bretagne, dans les milieux aisés, à 0 1. 454. Les autorités en matière d'alimentation estiment qu'elle devrait être de 01.568 et plus, ce qui correspond à une augmentation d'environ $100 \%$ pour la consommation journalière par tête si on prend le chiffre le plus bas, ou de $150 \%$ si on prend le chiffre fixé par les autorités en matière d'alimentation pour la eonsommation optimum. Pour produire 0 1. 568 (1 pinte) de lait par jour, il faudrait, ou que le rendement annuel moyen par vache passe de 2.044 litres à 4.497 litres, ou que le nombre des vaches laitières soit doublé, ou que les deux moyens soient combinés. Etant donnée la superficie relativement faible de la Grande-Bretagne, il est évident qu'une plus grande efficacité serait nécessaire dans toutes les branches de l'industrie Iaitière.

K. étudie ensuite les différents points du problème : qualité du lait ; rendement par vache; augmentation du nombre de vaches laitières ; adaptation des fermes laitières; augmentation des engrais; augmentation de la superficie réservée à la laiterie et augmentation de la dimension de l'unité laitière individuelle. Il estime qu'une augmentation de la production d'environ $30 \%$ en lait liquide pourrait être facilement obtenue; mais que les 70 autres pour cent ne seraient que très difficilement obtenus et très lentement.

Le problème de la production ne serait, du reste pas en pratique, le plus difficile à résoudre; celui de l'éducation du public serait beaucoup plus ardu. Vous pouvez fournir du lait au consommateur, mais vous ne pouvez pas l'obliger à le boire contre sa volonté en raison d'une opinion préconçue.

C. WoLF.

La laiterie finlandaise. Svenska Mejeritidningen. $30^{\mathrm{e}}$ année, no 4, 22 janvier 1938, p. 38-39.

La laiterie finlandaise s'est beaucoup développée depuis 1919, époque 